Artigo

\title{
Turismo Cultural y Policy Network: caracterización del modelo de negocio en la comuna de Camarones, Región de Arica y Parinacota, Chile
}

Turismo Cultural e Policy Network:

caracterização do modelo de negocio no municipio de Camarones, Região de Arica e Parinacota, Chile

Cultural Tourism and Policy Network: characterization of the business model in the commune of Camarones, Region of Arica and Parinacota, Chile

Tourisme culturel et policy network: caractérisation du modèle d'entreprise dans la commune de Camarones, Région d'Arica et de Parinacota, Chili

\footnotetext{
${ }^{1}$ Licenciado en Administración de Empresas por la Universidad Arturo Prat, Iquique, Chile, posgraduado en Diseño, Gestión y Dirección de Proyectos por la Universidad Europea del Atlántico, Santander, España. 
Resumen

\section{Resumo}

El artículo expone los hallazgos de una investigación destinada a estudiar la situación de la actividad turística cultural, desde la perspectiva de los modelos de negocio de la red empresarial como integrante del policy network de la comuna de Camarones, en tantos insumos para una propuesta de elaboración de políticas públicas de gobiernos locales. Para tales efectos se exponen las distinciones del turismo cultural, de los modelos de negocios, así como también su relación con el Plan de Desarrollo Comunal (Pladeco). La metodología de investigación empleada fue de tipo exploratorio cuantitativo y las categorías de análisis se construyeron basándose en las variables del modelo de negocios Canvas Business Model, herramienta de gestión estratégica que permite evaluar el diseño de un negocio en cuanto a su oferta, los clientes, su infraestructura, y la situación financiera del negocio, con el fin de observar deficiencias y analizar el valor entregado. Como resultado se pudo establecer una caracterización de la red de empresas que operan en la comuna de Camarones, así como una evaluación general del proceso de diseño de los modelos de negocios en cuanto al turismo cultural. Finalmente se concluye que los factores prioritarios a desarrollar por parte de las empresas turísticas de la comuna son básicamente tres: a) producto turístico cultural, b) asociatividad de la red c) diseño del modelo de negocio asociativo, aspectos que pueden ser abordados en la elaboración de futuros planes de desarrollo comunal.

Palabras Clave: Policy Network; Turismo Cultural; Modelo de Negocio; Canvas Business Model; Comuna de Camarones-Chile.

O artigo expõe os resultados de uma pesquisa destinada a estudar a situação da atividade turística cultural a partir da perspectiva dos modelos de negócios da rede de empresas como membro da rede de políticas da comuna de Camarones, em tantos insumos para uma proposta de elaboração de políticas públicas dos governos locais. Para tais efeitos são expostas às distinções do turismo cultural, dos modelos de negócios, assim como sua relação com o Plano de Desenvolvimento Comunitário (Pladeco). A metodologia de pesquisa utilizada foi exploratória quantitativa e as categorias de análise foram construídas com base nas variáveis do Modelo de Negócio Tela, uma ferramenta de gestão estratégica que permite avaliar o desenho de um negócio em sua oferta, clientes, infra-estrutura e a situação financeira do negócio, a fim de observar as deficiências e analisar o valor entregue. Como resultado, foi possível estabelecer uma caracterização da rede de empresas que operam na comuna de Camarones, bem como uma avaliação geral do processo de concepção de modelos de negócios, em termos de turismo cultural. Finalmente, conclui-se que os fatores prioritários a serem desenvolvidos por das empresas de turismo da comuna são basicamente três: a. produto turístico cultural, b. associatividade da rede c. modelo empresarial associativo, aspectos que podem ser abordados na elaboração de futuros planos de desenvolvimento comunitário.

Palavras-Chave: Policy Network; Turismo Cultural; Modelo de Negócios; Canvas Business Model; Comuna de Camarones-Chile. 
Abstract

Resumé
The article presents the findings of research aimed at studying the situation of the cultural tourism activity from the perspective of the business models of the entrepreneurial network as a member of the policy network of the commune of Camarones, as inputs for a proposal for the elaboration of public policies of local governments. For such purposes, the distinctions of cultural tourism, business models as well as their relationship with the Communal Development Plan (Pladeco) are presented. The research methodology used was quantitative exploratory and the categories of analysis were constructed based on the variables of the Canvas Business Model, a strategic management tool that allows evaluating the design of a business in terms of its offer, customers, infrastructure, and financial situation of the business, in order to observe deficiencies and analyse the value delivered. As a result, it was possible to establish a characterization of the network of businesses operating in the commune of Camarones as well as a general evaluation of the process of designing business models, in terms of cultural tourism. Finally, it is concluded that the priority factors to be developed the tourism enterprises of the commune are basically three: a) cultural tourism product, b) associativity of the network, c) design of the associative business model, aspects that can be addressed in the elaboration of future communal development plans.

Key words: Policy Network; Cultural Tourism; Business Model; Canvas Business Model; Commune of Camarones-Chile.

L'article expose les résultats d'une enquête destinée à étudier la situation de l'activité touristique culturelle du point de vue des modèles d'affaires du réseau d'entreprises en tant que membre du réseau politique de la commune de Camarones, en autant d'apports pour une proposition d'élaboration de politiques publiques des gouvernements locaux. Pour ces effets, les distinctions du tourisme culturel, des modèles d'affaires ainsi que sa relation avec le Plan de Développement Communal (Pladeco) sont exposés. La méthodologie de recherche utilisée était quantitative exploratoire et les catégories d'analyse ont été construites sur la base des variables du Canvas Business Model, un outil de gestion stratégique qui permet d'évaluer la conception d'une entreprise en termes d'offre, de clients, d'infrastructure et de situation financière de l'entreprise, afin d'observer les déficiences et d'analyser la valeur fournie. Il a ainsi été possible d'établir une caractérisation du réseau d'entreprises opérant dans la commune de Camarones ainsi qu'une évaluation générale du processus de conception de modèles d'entreprise, en termes de tourisme culturel. Finalement, on conclut que les facteurs prioritaires à développer des entreprises touristiques de la Commune sont essentiellement au nombre de trois: a) développement du produit touristique culturel, b)associativité du réseau c) modèle d'entreprise associatif, aspects qui pourront être abordés dans l'élaboration des futurs plans de développement communaux.

Mots Clés: Policy Network; Tourisme Culturel; Modèle d'Entreprise; Canvas Business Model; Commune de Camarones-Chile. 


\section{Introducción}

El turismo cultural desempeña un rol fundamental en las comunidades porque les permite dar a conocer, preservar y disfrutar de sus identidades en forma sostenible. Para los gobiernos locales, el lograr incluirlo en las políticas públicas como actuación del Estado en el ámbito del turismo (Lourenço \& Moreira, 2014), como eje estratégico para el desarrollo, supone un gran desafío debido a que mientras el turismo se ha considerado históricamente como una actividad principalmente económica, la cultura por su parte, constituye una riqueza que no puede considerarse en su totalidad un producto clásico de consumo. Sin embargo, Silva y Sanchez (2012) plantea también las posibilidades de reorganización local, considerando también, la visión del gobierno municipal respecto de los posibles impactos que el turismo puede traer al interior de la vida comunitaria.

Aun con la complejidad que se presenta al intentar establecer una definición conceptual y operativa del turismo cultural, es necesario definir los conceptos que lo componen de manera de sentar una base de distinciones con el objeto de analizar los modelos de negocios que operan en el área de estudio.

El concepto cultura en su definición institucional de la Unesco lo define como un "conjunto de rasgos distintivos, espirituales y materiales, intelectuales y afectivos que caracterizan una sociedad o un grupo social. Ello engloba, además de las artes y las letras, los modos de vida, los derechos fundamentales al ser humano, los sistemas de valores, las tradiciones y las creencias". (Unesco, 1982: 1). Por su parte, para la Organización Mundial de Turismo (OMT) el turismo "comprende las actividades que realizan las personas durante sus viajes y estancias en lugares distintos al de su entorno habitual, por un período de tiempo consecutivo inferior a un año, con fines de ocio, por negocios y otros motivos" (OMT, 1995: 1). Asimismo, la OMT aporta una definición de turismo cultural de carácter técnico y lo precisa como:

El movimiento de personas debido esencialmente a motivos culturales como viajes de estudio, viajes a festivales $u$ otros eventos artísticos, visitas a sitios o monumentos, viajes para estudiar la naturaleza, el arte, el folklore, y las peregrinaciones"; y otra forma de carácter más general: "todos los movimientos de personas para satisfacer la humana necesidad de diversidad, orientados a elevar el nivel cultural del individuo, facilitando nuevos conocimientos, experiencias y encuentros. (OMT, 1985: 1).

Por otra parte, de acuerdo con Andrade (2000), en su libro Turismo, fundamentos e dimensões, el autor define el turismo cultural como:

A experiência humana e todas as suas manifestações se constituem em fator cultural, mas, por coerência e respeito às finalidades que são meios classificatórios do turismo, a expressão turismo cultural possui conotação restritiva e abrange exclusivamente as atividades que se efetuam através de deslocamentos para a satisfação de objetivos de encontro com emoções artísticas, científicas, de formação e de informação nos diversos ramos existentes, em decorrência das próprias riquezas da inteligência e da criatividade humanas. (Andrade, 2000:71). 
Dado lo anterior podemos sintetizar una definición más sucinta para englobar las definiciones que nos anteceden: el Turismo Cultural es un subsector socioeconómico que centra sus esfuerzos en los atractivos derivados de los recursos basados en el patrimonio cultural y se vincula con el bagaje cultural de los habitantes de un determinado destino turístico. Así también Gagliardi (2015) agrega incluso que el mantenimiento del patrimonio cultural es condición para la existencia de la actividad turística de un destino, aun cuando allí se desarrollen otras modalidades de turismo donde la cultura no sea lo central.

Por su parte, el modelo de negocio como concepto se masificó a fines de los 90' producto de la globalización y de la influencia de las tecnologías de información. En este periodo las cadenas de valor empresarial cambiaron de forma, aumentó la competencia, emergieron nuevos canales de distribución y los clientes se empoderaron al contar con una mayor información, surgieron así nuevas formas de hacer negocios. Un segundo momento de importancia fue el intento de formalización de los modelos de negocios en la primera década de este siglo. En 2009 basado en un proceso de investigación y experiencia real, Alexander Osterwalder e Ives Peigner publicaron la obra "Generación de modelos de negocios" (Canvas Business Model Generation). Según estos autores "Un modelo de negocio describe las bases sobre las que una empresa crea, proporciona y capta valor" (Osterwalder \& Peigner, 2009: 17). Esta herramienta de gestión estratégica permite evaluar el diseño de un negocio en cuanto a su oferta, los clientes, su infraestructura, y la situación financiera del negocio, con el fin de observar deficiencias y analizar el valor entregado a sus clientes

El objetivo de este artículo es caracterizar el modelo de negocios de turismo cultural de la red empresarial de la comuna de Camarones orientado a constituir una propuesta de insumo para la formulación del Plan de Desarrollo Turístico, en tanto política pública comunal de la zona. Para ello dispusimos del método Canvas Business Model (CBM) (Osterwalder \& Peigner, 2009), como marco de referencia y análisis para una caracterización particular. En este sentido, la investigación - de tipo exploratoria cuantitativa - estuvo orientada a la profundización de un área de la epistemología turística poco explorada en el territorio de estudio.

Para este propósito, en el primer apartado se incluyen las principales características de la comuna de Camarones en cuanto al turismo cultural y de su red empresarial. En el apartado Metodología se entrega una breve descripción del CBM y el método de análisis de recogida de información de la red empresarial. Las Consideraciones Finales exponen los puntos críticos que pueden ser considerados en la elaboración de políticas públicas y futuras investigaciones del área de estudio.

\section{Turismo cultural en la comuna de Camarones}

Por su propia naturaleza, Camarones, unas de las cuatro comunas de la región de Arica y Parinacota, es un destino que atrae al turista cultural, ya que su ubicación, su conformación geográfica y su cultura ancestral le otorgan características excepcionales. La comuna rural de Camarones se ubica en la franja sur de la región de Arica y Parinacota, a su vez, delimita al norte con Perú, al este con Bolivia, al oeste con el océano Pacífico y al sur con la región de Tarapacá, lo que la convierte en una de las principales vías de acceso al país. 
Este territorio cuenta con diversos atractivos naturales Turísticos relacionados con elementos del relieve y del paisaje, así como también culturales asociadas a bienes patrimoniales y expresiones tradicionales que permiten desarrollar las diversas manifestaciones del turismo cultural a lo largo de una zona que presenta altitudes que van de $\operatorname{los} 0$ a los $4500 \mathrm{~m} / \mathrm{s} / \mathrm{n} / \mathrm{m}$ lo cual permite que se presenten tres áreas bien definidas, costa, meseta altiplánica y altiplano. La población indígena posee una significativa representación, tanto desde el punto de vista demográfico como desde su identidad, tradiciones e historia, según datos del Instituto Nacional de Estadísticas (INE) "la etnia aymara es la que destaca y se encuentra presente en toda la comuna" (INE, 2002: 127).

El turismo cultural en la comuna de Camarones, se apoya básicamente en la existencia de sus atractivos turísticos compuestos por sus recursos naturales y culturales los cuales permiten desarrollar el turismo cultural, que derivan de la particularidad geográfica y cultural de su territorio, por lo cual se relacionan directamente con la identidad local. Según OEA un atractivo turístico "es todo lugar, objeto o acontecimiento de interés turístico" (OEA, 1978: 4) y de una forma más operativa el concepto de atractivo turístico lo define Navarro como "el conjunto de elementos materiales y/o inmateriales que son susceptibles de ser transformados en un producto turístico que tenga capacidad para incidir sobre el proceso de decisión del turista provocando su visita a través de flujos de desplazamientos desde su lugar de residencia habitual hacia un determinado territorio. Este último se transforma de esta manera en un destino turístico (Navarro, 2010:47).

Un grupo significativo de los atractivos de la comuna de Camarones son representativos del relieve, así como de los diferentes paisajes que estructuran su territorio. Estos a su vez, se constituyen en escenarios para la realización de prácticas, eventos tradicionales y ancestrales de sus habitantes, su implementación, para uso turístico, contribuyen a difundir el conocimiento a sus visitantes. Actualmente, se dispone de dos grandes fuentes documentales para la consulta del catastro de sus atractivos culturales, una de acuerdo con la clasificación de inventarios realizada por Servicio Nacional de Turismo (Sernatur) y otra por la Comisión Asesora de Monumentos Nacionales de la región de Arica y Parinacota. Estos catastros han sido de gran utilidad no solo para el turista, sino que además como una herramienta fundamental para los emprendimientos con un enfoque cultural en la comuna de Camarones.

Cabe señalar que ciertamente muchos de los atractivos están por descubrir; esto implica por una parte que la comuna presenta oportunidades futuras y por otra que las riquezas turísticas de esta zona son complementarias con las de otros territorios, por lo tanto, el turismo se transforma en un factor no solo de integración nacional y sino que también internacional, considerando la macro zona andina donde se ubica. El año 2012, Sernatur actualizó el catastro de atractivos turísticos en todo el país y está de acuerdo con la clasificación propuesta por la metodología OEA-CICATUR, de los 300 atractivos turísticos identificados 22 corresponde a la comuna de Camarones (Sernatur, 2002).

El poder poner en valor los atractivos naturales y culturales, significa un desafío y una oportunidad al mismo tiempo, por un lado, se deben establecer las acciones para protegerlos, ya que su pérdida, por grave alteración o daño, es irrecuperable y por otra parte constituirlos en la plataforma donde se diseñaran los emprendimientos turísticos, que le darán la sustentabilidad que le permitan conservarlos, ya que su función esencial es formar parte del patrimonio y la riqueza colectiva. Por esto, el turismo cultural en la comuna de Camarones, puede ayudar a mejorar las condiciones de vida de las comunidades más vulnerables, reducir la pobreza, proteger el medioambiente y ampliar las 
relaciones interculturales, convirtiéndose así en un catalizador de progreso y crecimiento. Lo anterior queda en evidencia en las consideraciones de unos de los documentos de política pública más relevantes del territorio: el Plan de Desarrollo Comunal de Camarones 2012-20161 y se refiere al turismo de la siguiente manera:

\begin{abstract}
El turismo por medio de la capacitación a los comuneros en temas relacionados a su historia, identidad y vinculados a servicios turísticos. Dentro del eje productivo, el fomento al turismo se proyecta como una fuente de ingresos y como la posibilidad para el crecimiento futuro de la comuna, para los habitantes de Camarones el contar con condiciones para desarrollar esta actividad es de suma importancia, ya que evidencian que dentro de las fortalezas con que cuentan, está su patrimonio arqueológico, histórico y cultural. La habilitación de rutas turísticas, la reparación de iglesias y la puesta en valor de sitios arqueológicos, son posibles proyectos que cuentan con la aprobación de la comunidad. (Pladeco 2012-2016, 2012: 13).
\end{abstract}

Por lo tanto, el avance del turismo cultural en la comuna de Camarones es un aspecto central para un desarrollo inclusivo de su comunidad permitiendo construir una imagen plural, puesto que como hemos visto anteriormente, la asociación entre cultura y turismo, trasciende a su importancia económica y productiva, al potenciar actividades que también aportan otros beneficios como los son los sociales y ambientales. Además, su red empresarial como parte del entramado de política pública (policy network) otorgan los insumos necesarios - aunque no suficientes - para contribuir al diseño de políticas de gobierno local, como el Pladeco e instrumentos más específicos como es el caso de los Planes de Desarrollo Turístico de la comuna (Pladetur). De esta forma, la red empresarial - en cuanto actores relevantes del turismo - funcionan como una red de política pública (Godoy, 2019) que contribuye con las informaciones necesarias para la elaboración de estos instrumentos de planificación del gobierno local.

\title{
2. Metodología
}

La metodología del presente trabajo se basa en las propuestas de análisis del Canvas Bussines Model (Osterwalder \& Peigner, 2009). Cada elemento proporciona una categoría de análisis capaz de configurar la forma en que se desempeñará el negocio en relación a su grupo de interés (stakeholder). De esta forma, cada categoría de análisis del modelo proporciona una información cuantitativa.

\footnotetext{
${ }^{1}$ Según CEPAL, el Plan de Desarrollo Comunal (PLADECO) es un instrumento indicativo que cada municipio debe tener según la Ley Orgánica Constitucional de Municipalidades y debe orientar el desarrollo de la comuna. Tiene una duración mínima de cuatro años y debe ser aprobado por el concejo municipal. Se considera un instrumento mínimo para la gestión municipal.
} 


\subsection{El Canvas Business Model (CBM)}

El CBM es una herramienta de gestión estratégica para el diseño de nuevos negocios o para representar modelos de negocios ya en marcha. Como se puede apreciar en la figura 1, es un gráfico visual, que básicamente describe la oferta, la infraestructura, los clientes y las finanzas de un negocio. En general, la metodología del CBM, por ser un modelo visual que se desarrolla en un lienzo, es un muy buen punto de partida y ayuda al pensamiento estratégico de las empresas, a través de un sistema organizado que permite distinguir las relaciones entre los distintos aspectos de un modelo de negocios según lo mostrado en la figura 1. Sin embargo, esta metodología no garantiza o anticipa algunos aspectos operativos de la empresa, solo ofrece una noción de ellos.

Figura 1: Modelo de negocios propuesto por Osterwalder (2009)

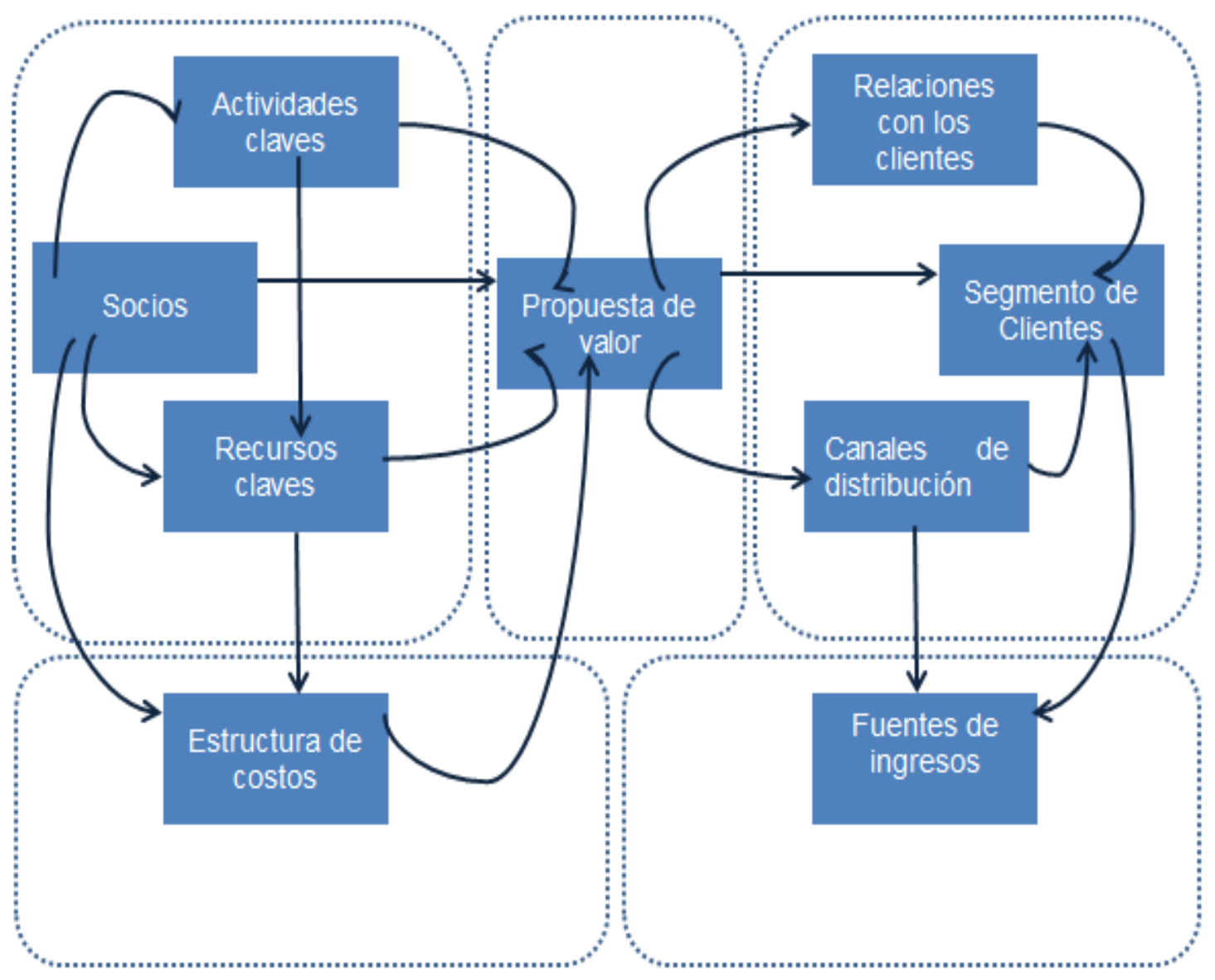

Fuente: Elaboración propia basada en el CBM. www.businessmodelgeneration.com 
Los elementos que lo conforman el BCM son los siguientes: Segmentos de clientes: El objetivo es agrupar a los clientes con características homogéneas en segmentos definidos y describir sus necesidades. Propuesta de valor: Se define el valor creado para cada segmento de clientes, describiendo los productos y servicios que se ofrecen a cada uno. Canales de distribución: Se resuelve la manera en que se establece contacto con los clientes. Cada producto o servicio tendrá su canal de su distribución. Relaciones con el cliente: Se identifica la forma y cuáles son los recursos que utilizan para mantenerse en contacto con los clientes. Fuentes de ingresos: Se determina el aporte monetario que hace cada segmento de clientes, de modo de saber de dónde vienen las entradas económicas. Recursos clave: Se establecen cuáles son los recursos clave que intervienen para que la empresa tenga la capacidad de entregar su propuesta de valor. Actividades clave: Se definen las actividades necesarias para entregar la propuesta de valor. Se distinguen las actividades de producción, las de solución de problemas, las de creación de plataformas y construcción de redes. Asociaciones claves: Se analizan las alianzas estratégicas entre empresas u organizaciones. Estructura de costos: Se especifican las estrategias de costos del negocio que pueden ser de entrega de beneficios, de volumen o mixta.

\subsection{Análisis del CBM en la Comuna de Camarones}

Para el presente estudio se ha utilizado un diseño de investigación de tipo exploratorio cuantitativo, luego que revisada la bibliografía correspondiente se determinó que en la comuna de Camarones existía escasa información acerca del turismo cultural y su relación con los modelos de negocios, además han sido escasamente investigados y las indagaciones han sido muy acotadas. Este tipo de investigación nos permitirá esclarecer y delimitar el objetivo de investigación y asimismo analizar las variables de interés, que son la asociatividad y la propuesta de valor de los modelos de negocios turísticos que están operando actualmente en la comuna de Camarones. La estrategia adoptada para el presente trabajo será una investigación de campo para responder al objetivo planteado. La información será recopilada tanto de fuentes primarias a través del cuestionario aplicado a los emprendedores de la comuna de Camarones y secundaria respecto a la revisión bibliográfica pertinente al estudio.

A modo de antecedentes, se tiene que según los datos recolectados en el censo del año 2002 realizado por el Instituto Nacional de Estadísticas (INE), la comuna de Camarones posee una población de 1.220 habitantes (distribuidos en 658 viviendas), de los cuales 475 son mujeres y 745 son hombres. La comuna de Camarones acoge al 0,6\% de la población total de la región de Arica y Parinacota, y su población es $100 \%$ rural. El $61,6 \%$ de la misma se reconoce como indígena. Sin embargo, en este estudio censal no existe información de los emprendedores por sectores productivos.

Para el presente estudio la población estará conformada por empresas formales pertenecientes a la comuna de Camarones que cuenten con una "declaración de inicio de actividades" ante el Servicio de Impuestos Internos de Chile. Además se adoptan en esta categoría al emprendimiento que componga la red empresarial turística de la zona tales como empresas de hospedaje, alimentación, transporte de pasajeros y tour operadores. La muestra corresponde a 30 empresas del rubro estudiado. 
El tipo de muestreo utilizado es del tipo no probabilístico, intencional, donde se seleccionó una muestra por conveniencia de la población, la cual no se realizó sobre la base de un criterio de aleatoriedad, sin embargo, por medio del procedimiento del punto de saturación se procuró garantizar la representatividad de la muestra.

Para la obtención de datos de fuentes primarias, fue necesaria definir variables en base con las categorías definidas en BMC para luego construcción de un cuestionario con preguntas abiertas y cerradas. Este sirvió como instrumento de evaluación para obtener una aproximación general de la situación de la red empresarial, permitió describir factores críticos de la mecánica utilizada en el diseño de sus modelos de negocios.

Este cuestionario estuvo dirigido al dueño, gerente o administrador encargado de la toma de decisiones dentro de la empresa y aplicado de forma presencial entre marzo a junio de 2016. Para determinar la aplicabilidad y pertinencia del cuestionario de medición, fue necesario llevar a cabo una prueba piloto a 5 entrevistados, con el objeto de asegurar la comprensión de las preguntas y realizar cambios oportunos de los factores a medir en la estructura definitiva. La estructura del cuestionario se dividió en 4 secciones: Sección I Antecedentes Generales, Sección II: del Diseño de Modelos de Negocios. Sección III: Elementos del Diseño de Modelos de Negocios. Sección IV: del Turismo Cultural.

\section{Resultados}

\subsection{Sección I: Antecedentes generales}

Las empresas que componen la red empresarial tienen una antigüedad promedio de 9,6 años realizando actividades de emprendimiento, un 73,3\% de ellos han recibido estudios superiores, 23,3\% enseñanza media, mientras que un 3,3\% cuentan con estudios básicos. Un $90 \%$ señala que las tareas de administración de sus empresas son realizadas por ellos mismos. Asimismo, se tiene que 93,3\% de los emprendimientos corresponden a microempresas y un $6,7 \%$ al sector de pequeñas empresas. Los rubros corresponden a empresas de transporte de pasajeros, servicio de hospedaje, servicios de camping, restaurantes, ganaderos y agricultores. En cuanto a la empleabilidad 3,6 personas (en promedio) trabajan directamente en estas empresas.

Dado lo anterior se puede establecer una caracterización general de la red de empresas que operan en la comuna de Camarones. Por una parte tenemos que los emprendedores consultados pertenecen directa e indirectamente al sector turístico cultural, casi en su totalidad son empresas familiares, presentan una estructura organizacional más bien plana y compacta (Köller, 2014). La administración es empírica y es ejercida por sus propios dueños, los cuales dominan el rubro y las prestaciones que ofrecen. El capital humano es importante en los negocios, esto se refleja en la alta tasa de este insumo en la composición de la cual están conformados el negocio. 
Además, se presentan dificultades para contratar trabajadores calificados que realizan las actividades clave del negocio que le permiten escalar a un mayor tamaño de empresa.

\subsection{Sección II: del diseño de modelos de negocios}

En relación con el diseño de modelos de negocio un 36,7\% han utilizado herramientas para el diseño de negocios, mientras que el $63,3 \%$ no lo ha hecho. Por otra parte, $10,5 \%$ recurre a asesoría externa y un $89,5 \%$ lo hace por cuenta propia. La motivación para llevar a cabo este proceso ha sido en $68,4 \%$ para solicitar subsidios a instituciones de gobierno, en un $21,1 \%$ solicitar financiamiento a instituciones financieras privadas, y un 10,5\% como herramienta estratégica para su negocio. Cabe señalar que las herramientas que utilizan son los planes de negocios y los formularios solicitados por los organismos públicos, para postular a programa de fomento y subsidios de gobierno en forma individual y no asociativa. Asimismo, solo 6,7\% de ellos afirman que han utilizado la herramienta diseño de negocios CANVAS. Del grupo que no utilizaba herramientas, la razones seleccionadas para no adoptarlas, corresponde en un $68,4 \%$ a su alto costo, un $21,1 \%$ porque no encuentra en el mercado la que necesita y un $10,5 \%$ porque no encuentra que sea necesario utilizarla. De lo anterior se desprende que diseño de modelos de negocios es realizado internamente si la contratación de servicios de consultoría y no es utilizado como herramienta estratégica sino cuando se necesita postular a un programa de gobierno.

\subsection{Sección III: Elementos del diseño de modelos de negocios}

Proposición de valor: respecto a la definición de la propuesta de valor, al preguntar que entrega el negocio a sus clientes, un $83,3 \%$ señala que satisface una necesidad y un $16,7 \%$ aporta un beneficio. En cuanto a los que buscan los clientes, un $76,7 \%$ señala que disminuir sus costos, un $10 \%$ que le facilite la vida, un $6,7 \%$ excelencia en el producto o servicio, mientras que un 3,3\%, algo inédito. Por su parte, un $83 \%$ cree que la percepción de los clientes de la propuesta de valor es buena, un 6,7\% es muy buena y un $10 \%$ regular. Cuando se le pregunta respecto a cuan bien sus clientes están siendo servidos por sus competidores, por medio de la propuesta de valor de ellos en un 56,7\% considera que es similar, un $20 \%$ que es mejor y $23,3 \%$ que es menor. Respecto a si complementa su propuesta de valor en alianza con otro proveedor un 6,7\% lo hace mientras que $93,3 \%$ afirma que no lo realiza. .

Creación de valor: un 36,7\% afirma que tiene socios claves, en tanto que un 63,3\% contesto que no tenía. Además, un 30\% declara que es participante activo de una asociación gremial. En cuanto a las actividades claves de los modelos de negocios, el mayor énfasis está puesto en las actividades de desarrollo de productos o servicios con un 55,7\%, luego las de desarrollo de clientes con un 26,8\% y por último en las de creación de redes con un 17,5\%. La importancia que los emprendedores le dan a los recursos claves queda valorada de la siguiente forma: recursos físicos con un 30,8\%, recursos intelectuales con un $16 \%$, recursos humanos con un $31,7 \%$, y recursos financieros con $21,5 \%$. 
Captura de valor: los flujos de ingreso del negocio provienen en un 36,7\% de la venta directa y un $63,3 \%$ por el arriendo de los bienes y servicios. Por otro lado Un 6,7\% no ha solicitado financiamiento externo mientras que un $96,3 \%$ de los emprendedores lo ha hecho. Respecto a donde los ha solicitado un $53,3 \%$ en la banca privada, un $23,3 \%$ en instituciones gobierno y un $16,7 \%$ en otra fuente y señalan que este financiamiento es para ser usado para la adquisición de bienes de capital (56,7\%), para capital de trabajo $(26,7 \%)$ y para mejorar la infraestructura de sus negocios $(16,7 \%)$. Por su parte, respecto a la estrategia de costos los emprendedores utilizan la alternativa de bajo costo con un 73,3\%, la de beneficios con un $20 \%$ y un $6,7 \%$ usa una estrategia mixta. Asimismo, un $75 \%$ alcanza economías de escala un $15 \%$ economías de alcance. Un $67 \%$ de los encuestados utiliza mecanismo de control para sus costos.

Una de las debilidades identificada en la construcción de los modelos de negocios de los emprendedores encuestados, corresponde a ofertas poco desarrolladas, existe una insuficiente identificación de la propuesta de valor para sus clientes, ya que a pesar de tener un adecuado conocimiento del negocio en el cual participan y de los atractivos de la comuna de Camarones, se ofrecen productos y servicios genéricos, centrados en el conocimiento productivo, sin análisis de las necesidades y deseos del cliente, asimismo los productos o servicios se enfocan hacia un mercado masivo, que es compartido por el resto de los competidores o participantes. Sin una propuesta asociativa.

La integración empresarial se muestra débil, esto se manifiesta en la escasa capacidad de asociatividad como lo muestra el bajo nivel de alianzas entre operadores de la red empresarial turística de la comuna y de la región que pertenece (Arica y Parinacota) luego que un $70 \%$ reconoce que no trabaja asociativamente. Esto tiene como consecuencia bajo poder de negociación, tienen dificultad para conseguir proveedores de envergadura, además la propuesta de valor del negocio no es establecida con una visión asociativa, se desaprovecha la oportunidad de economías de escala. Esta situación coincide con el diagnóstico de la Política Regional de Turismo Arica y Parinacota 2012-2016 (Godoy, 2019), donde el diagnóstico de entregada observa un bajo nivel de asociatividad empresarial en el sector del turismo regional. Otro aspecto central es una escasa penetración en los canales de comercialización turística por lo que se observa falta de relaciones intersectoriales que potencian el desarrollo turístico.

Para su crecimiento, los emprendedores buscan financiamiento para sus empresas, para realizar inversiones de bienes de capital, para mejorar infraestructura o contar con el capital de trabajo que aseguren sus operaciones que mejorará su posición competitiva en los mercados, en este sentido el emprendedor tienen acceso restringido a fuentes de financiamiento de la banca privada. No obstante la mayoría de las empresas ha hecho uso de los subsidios de diversas instituciones públicas, por medio de sus instrumentos de fomento productivo solo en forma individual y no asociativa.

\subsection{Sección IV: del turismo cultural}

Un $85 \%$ de los encuestados considera que los atractivos de la comuna de Camarones pueden consolidarla como un destino turístico. A su vez, solo un $31 \%$ incorpora alguna manifestación turística en su oferta. Aunque una gran proporción ven una oportunidad en sus atractivos culturales, no son utilizados en la elaboración de sus servicios y productos. 


\section{Consideraciones Finales}

La red empresarial de la comuna de Camarones puede desarrollar el turismo cultural, porque dispone de abundantes atractivos (naturales y culturales) y un modelo de negocio potencial de una comunidad que aspira emprender con esta actividad. Esto se sintoniza con lo declarado en el Plan de Desarrollo Comunal 2012-2016 (Pladeco) que reconoce al turismo como eje estratégico de fomento productivo.

Sin embargo existe una baja competitividad de las empresas turísticas de la zona, por lo que los factores prioritarios a desarrollar para la mejora de este aspecto son básicamente tres: a) producto turístico, b) asociatividad de la red y c) diseño del modelo de negocio. Además, se ha podido constatar que existe un bajo porcentaje (25\%) de emprendedores que aprovechan los atractivos naturales y culturales para sus emprendimientos, lo que también se puede considerar como un factor limitante de la competitividad. Por otra parte, la comunidad deberá atenuar las eventuales externalidades que arroja esta actividad como por ejemplo la pseudo indigenización, la precarización de empleos, el daño al medioambiente y a los sitios arqueológicos.

Para llevar adelante los emprendimientos con un enfoque turístico cultural, implica para la red empresarial (policy network) involucrada y el gobierno local, un esfuerzo sustancial de planificación con visión estratégica que permitan mejorar su competitividad, sobre todo en la mejora de la asociatividad, el perfeccionamiento del capital humano y de la articulación de productos turísticos que propendan al desarrollo sustentable de sus localidades. Por medio de la elaboración de políticas (policies) integradas que estimulen un contexto democrático y la participación de los demás actores relevantes del territorio (Silva \& Sanchez, 2012 ; Ortolano, 2012), además del compromiso de una política efectiva que fomente la sustentabilidad turística, la puesta en valor y la conservación del patrimonio cultural de la comuna de Camarones. 


\section{Referencias Bibliográficas}

Andrade, José. (2000). Turismo. Fundamentos e dimensões. São Paulo: Ática.

Gagliardi, Clarissa (2015). O patrimônio cultural como atrativo turístico. En Netto y Ansarah. Produtos turísticos e novos segmentos de mercado. São Paulo: Manole.

Godoy, Iván (2019). La participación social en el proceso de elaboración de la Política Regional de Turismo de Arica y Parinacota 2012-2016, Revista Reforma y Democracia, 74.

INE (2002). Estadísticas sociales de los pueblos indígenas en Chile. CENSO 2002. Instituto Nacional de Estadísticas. Chile.

Köhler, André Fontan. (2014). Políticas Públicas de Cultura e Turismo: a transformação da cidade intramuros de York em precinto Urbano-turístico com alto grau de turistificação. Revista Gestão \& Políticas Públicas, 4(1), 154-175. Accedido en 4 de Febrero de 2020, de: https://www.revistas.usp.br/rgpp/article/view/114320

Lourenço, Fábio., \& Moreira, José M. (2014). A Lógica da Governance - Governação/Governança nas Políticas Públicas de Turismo. Revista Gestão \& Políticas Públicas, 4(1), 22-44. Accedido en 4 de Febrero de 2020, de: https://www.revistas.usp.br/rgpp/article/view/114309

Navarro, Diego. (2010): Imagen Turística Argentina. Política turística como política exterior. Mendoza, Editorial de la Universidad del Aconcagua.

OEA (1978). Metodologia de Inventario Turístico CICATUR/OEA. Organización de los Estados Americanos. Accedido en 4 de Febrero de 2020, de: https://turgeoyanedel.files.wordpress.com/2011/12/guia_metodologia_cicatur_modificada_rfs2011. pdf

OMT (1985). The state's rolein protecting and promoting culture as a factor of tourism development and the Proper Use and Exploitation of the National Cultural Heritage of Sites and Monument for Tourism. Accedido en 4 de Febrero de 2020, de: https://www.eunwto.org/doi/abs/10.18111/9789284409075

OMT (1995). UNWTO technical manual: Collection of Tourism Expenditure Statistics. Accedido en 4 de Febrero de 2020, de: https://www.e-unwto.org/doi/book/10.18111/9789284401062

Ortolano, Fabio. (2012). Turismo e Hospitalidade: um olhar para a juventude nas paradas do orgulho LGBT. Revista Gestão \& Políticas Públicas, 2(2), 314-330. Accedido en 4 de Febrero de 2020, de: https://www.revistas.usp.br/rgpp/article/view/97866

Osterwalder, Alexander., \& Peigner, Ives. (2009). Business Model Generation. Barcelona: Deusto.

Pladeco 2012-216 (2012) Plan de Desarrollo Comunal de Camarones. Camarones: RIMISP.

Silva, Alessandro,. \& Sanchez, Thais. (2012). Participación Política y Políticas Públicas: Estudio sobre las consecuencias psicopolíticas del Turismo en Paranapiacaba - Santo André/Sao Paolo, Brasil. Revista MEC-EDUPAZ, II Marzo. Accedido en 4 de Febrero de 2020, de: https://repositorio.unam.mx/contenidos/26848

UNESCO. (1982). Conferencia mundial sobre las políticas culturales. Accedido en 4 de Febrero de 2020, de: https://culturalrights.net/descargas/drets_culturals400.pdf

Recebido em 18/02/2020. Revisado em 30/04/2020. Aceito 12/05/2020. 